\title{
A NOTE ON FINITELY GENERATED IDEALS WHICH ARE LOCALLY PRINCIPAL
}

\author{
JAMES W. BREWER AND EDGAR A. RUTTER
}

\begin{abstract}
Let $R$ be a commutative ring with identity $1 \neq 0$ and let $A$ be a nonzero ideal of $R$. A problem of current interest is to relate the notions of "projective ideal", "flat ideal" and "multiplication ideal". In this note we prove two results which show that the maximal ideals containing the annihilator of $A$ can play an important role in determining the relationship between these concepts. As a consequence we are able to prove that a finitely generated multiplication ideal in a semi-quasi-local ring is principal, that a finitely generated flat ideal having only a finite number of minimal prime divisors is projective and that for Noetherian rings or semihereditary rings, finitely generated multiplication ideals with zero annihilator are invertible.
\end{abstract}

Our notation is essentially that of [4]. In particular, an ideal $A$ of $R$ is said to be a multiplication ideal if whenever $B$ is an ideal of $R$ with $B \subset A$, there exists an ideal $C$ of $R$ such that $B=A C$. There is one deviation from the notation of [4] and that is that we shall denote by $A^{\perp}$ the annihilator of an ideal $A$.

THEOREM 1. Let $A$ be a finitely generated multiplication ideal of $R$. If $A^{\perp}$ is contained in only finitely many maximal ideals, then $A$ is principal.

Proof. Let $M_{1}, \cdots, M_{n}$ be the maximal ideals of $R$ containing $A^{\perp}$. For $i$ between 1 and $n$, if $\prod_{j=1 ; j \neq i}^{n} M_{j} A \subseteq M_{i} A$, then $M_{i} A R_{M_{i}} \supseteq$ $\left(\prod_{j=1 ; j \neq i}^{n} M_{j} A\right) R_{M_{i}}=A R_{M_{i}}$. Since $A R_{M_{i}}$ is finitely generated and since $M_{i} R_{M_{i}}$ is the Jacobson radical of $R_{M_{i}}$, it follows from the Nakayama Lemma that $A R_{M_{i}}=(0)$. Thus, $A^{\perp} R_{M_{i}}=\left(A R_{M_{i}}\right)^{\perp}=R_{M_{i}}$, which contradicts the fact that $A^{\perp} \subseteq M_{i}$. Therefore, for $1 \leqq i \leqq n$, there exists $a_{i} \in$ $\left(\prod_{j=1 ; j \neq i}^{n} M_{j} A\right) \backslash M_{i} A$. Put $a=\sum_{i=1}^{n} a_{i}$. Then $a \in A \backslash A M_{i}, 1 \leqq i \leqq n$. Since $A$ is a multiplication ideal, $a R=A(a R: A)$ and therefore to complete the proof it suffices to see that $a R: A=R$. Let $M$ be a maximal ideal of $R$. If $M \notin\left\{M_{i}\right\}_{i=1}^{n}$, then $M \notin A^{\perp}$ and consequently $M$ does not contain ( $\left.a R: A\right)$. If $M \in\left\{M_{i}\right\}_{i=1}^{n}$, then we also have that $M \notin(a R: A)$ since $a \notin A M$.

Received by the editors March 15, 1971.

AMS 1969 subject classifications. Primary 1320; Secondary 1340.

Key words and phrases. Flat ideal, projective ideal, multiplication ideal, principal ideal.

(c) American Mathematical Society 1972 
It is known that an invertible ideal in a semi-quasi-local integral domain must be principal and Smith in [5, p. 1058] proves that a finitely generated multiplication ideal in a quasi-local ring is principal. Theorem 1 yields the following obvious generalization of these facts.

COROllary 1. A finitely generated ideal in a semi-quasi-local ring is a multiplication ideal if and only if it is a principal ideal.

We remark that since a finitely generated ideal is a multiplication ideal if and only if it is locally principal, it follows from [5, p. 1058] and Corollary 1 that a finitely generated flat ideal in a semi-quasi-local ring must be principal.

We remark also that Smith erroneously asserts in [5, Proposition A] that a finitely generated multiplication ideal whose annihilator is contained in the Jacobson radical must be flat. That this assertion is false may be seen easily since it fails to hold in any special primary ring, for example $Z / 4 Z$. However, one sees easily from Smith's results that if the annihilator of a finitely generated flat ideal is contained in the Jacobson radical, then the annihilator is (0). Moreover, a finitely generated multiplication ideal with annihilator (0) must be projective. In fact, such ideals are precisely the finitely generated ideals which are locally invertible. As Exercise 12 of $[1$, p. 179] shows, such an ideal need not be invertible and therefore, it is natural to ask for what rings such ideals are always invertible. We now show that the class of rings for which this is true is quite large. The following result is in the spirit of Endo [2, p. 287].

Proposition 1. Let $R$ be a ring and suppose that $S$ is a multiplicatively closed subset of $R$ consisting entirely of regular elements. If finitely generated locally invertible ideals of $R_{S}$ are invertible, then the same is true for $R$.

Proof. If $A$ is a finitely generated locally invertible ideal of $R$, then $A$ is a multiplication ideal and $A^{\perp}=(0)$. It follows that $A R_{S}$ is a finitely generated multiplication ideal of $R_{S}$ and that $\left(A R_{S}\right)^{\perp}=\left(A^{\perp}\right) R_{S}=(0)$. By hypothesis, $A R_{S}$ is invertible and therefore contains a regular element $a / s$, $a \in A, s \in S$. Thus, $a$ is a regular element of $R$ contained in $A$ and since $A$ is a multiplication ideal of $R$, there exists an ideal $B$ of $R$ such that $a R=A B$. It follows that $A$ is invertible.

COROLlaRY 2. If $R$ is Noetherian or semihereditary, then finitely generated locally invertible ideals of $R$ are invertible.

Proof. If $R$ is Noetherian, then the total quotient ring of $R$ is semiquasi-local. Apply Corollary 1 and Proposition 1.

If $R$ is semihereditary, then by [3, p. 113], the total quotient ring of $R$ is a von Neumann regular ring. But a finitely generated ideal in a von 
Neumann regular ring is principal, generated by an idempotent. Thus, in such a ring the only finitely generated locally invertible ideal is the ring itself. The result follows from Proposition 1.

We observe that our proof of the first part of Corollary 2 works in case $R$ is a ring with few zero-divisors-that is, a ring whose total quotient ring is semi-quasi-local. Also, we need only assume that $R$ is quasiregular-that is, a ring whose total quotient ring is a von Neumann regular ring - for the second part of our argument to apply.

Lemma 1. Let $A$ be an ideal of $R$ and assume that for each maximal ideal $M$ of $R$ with $M \supseteq A, A R_{M}=(0)$. Suppose further that there exists a finite number of prime ideals $P_{1}, \cdots, P_{n}$ of $R$ such that each maximal ideal $M$ of $R$ which contains $A^{\perp}$ contains some $P_{j}$. Then $A$ is principalgenerated by an idempotent.

Proof. Suppose that $A \subseteq \bigcap_{i=1}^{n} P_{i}$ and let $M$ be a maximal ideal of $R$. If $A \nsubseteq M$, then $A R_{M}=R_{M}$ and thus, $(0) \subseteq A^{\perp} R_{M} \subseteq\left(A R_{M}\right)^{\perp}=(0)$. Therefore, $A^{\perp} \subseteq M$ and $M$ contains $P_{j}$ for some $j$. But $P_{j} \supseteq \bigcap_{i=1}^{n} P_{i} \supseteq A$. Thus, $A \subseteq J$, the Jacobson radical of $R$ and $A=(0)$. Now assume that the notation is such that $A \subseteq \bigcap_{i=1}^{r} P_{i}$ and that $A \nsubseteq P_{r+1}, \cdots, P_{n}, r<n$. For $r+1 \leqq i \leqq n, A+P_{i}=R$; for suppose that there exists a maximal ideal $M$ of $R$ with $M \supseteq A+P_{i}$. Then $A R_{M}=(0)$, and since $A \nsubseteq P_{i}$, there exists $a \in A \backslash P_{i}$. Thus, we can find $s \in R \backslash M$ such that $s a=0 \in P_{i}$. This contradiction shows that $A+\left(\bigcap_{i=r+1}^{n} P_{i}\right)=R$ and so we can choose $a \in A, q \in \bigcap_{i=r+1}^{n} P_{i}$ such that $a+q=1$. If $M$ is a maximal ideal of $R$ and if $M \supseteq A$, then $(0) \subseteq a R_{M} \subseteq$ $A R_{M}=(0)$. If $M \notin A$, then as argued previously, $M \supseteq A^{\perp}$ and therefore contains $P_{j}$ for some $j$ between $r+1$ and $n$. Thus, $a \notin M$ and $R_{M} \supseteq A R_{M} \supseteq$ $a R_{M}=R_{M}$. It follows that $a R=A$, but we also have that $A R_{M}=\left(A R_{M}\right)^{2}=$ $A^{2} R_{M}$. Therefore, $A$ is a finitely generated idempotent ideal and consequently is generated by an idempotent.

We are now able to easily extend Theorem 3.3 of [6].

THEOREM 2. Let A be a finitely generated flat ideal of $R$. If $A$ has only a finite number of minimal prime divisors, then $A$ is projective.

Proof. Let $P_{1}, \cdots, P_{n}$ be the minimal prime divisors of $A$. Since $A$ is a finitely generated flat ideal, by [5, p. 1059], $A^{\perp} R_{M}=\left(A R_{M}\right)^{\perp}=(0)$ or $R_{M}$ for each maximal ideal $M$ of $R$. Moreover, $A \subseteq\left(A^{\perp}\right)^{\perp}$ and for each maximal ideal $M$ of $R$ with $M \supseteq\left(A^{\perp}\right)^{\lrcorner}, M \supseteq \sqrt{ }(A)=\bigcap_{i=1}^{n} P_{i}$. Thus, $M \supseteq P_{j}$ for some $j$ and by Lemma $1, A^{\perp}$ is principal. The result follows from [6, p. 508].

As an additional consequence of Lemma 1, we have the following result, also in the spirit of [2, p. 287]. 
THEOREM 3. If the Jacobson radical of $R$ contains a finite intersection of prime ideals, then finitely generated flat $R$-modules are projective.

Proof. Let $P_{1}, \cdots, P_{n}$ be prime ideals of $R$ with $\bigcap_{i=1}^{n} P_{i} \subseteq J$, the Jacobson radical of $R$. By [6, p. 508], it suffices to prove that cyclic flat $R$-modules are projective. Thus, let $R / A$ be such an $R$-module where $A$ is an ideal of $R$. If $M$ is a maximal ideal of $R$, then the sequence $0 \rightarrow A R_{M} \rightarrow$ $R_{M} \rightarrow(R / A) \otimes_{R} R_{M} \rightarrow 0$ is exact and by [1, p. 167], $(R / A) \otimes_{R} R_{M}$ is a projective $R_{M}$-module. Thus, $A R_{M}$ is principal generated by an idempotent, that is, $A R_{M}=(0)$ or $A R_{M}=R_{M}$. Moreover, if $M \supseteq A^{\perp}$, then $M \supseteq J \supseteq$ $\bigcap_{i=1}^{n} P_{i}$ and so $M \supseteq P_{j}$ for some $j$. By Lemma $1, A$ is principal generated by an idempotent and therefore $R / A$ is projective.

\section{REFERENCES}

1. N. Bourbaki, Algèbre commutative. Chaps. 1, 2, Actualités Sci. Indust., no. 1290, Hermann, Paris, 1961. MR 36 \#146.

2. S. Endo, On flat modules over commutative rings, J. Math. Soc. Japan 14 (1962), 284-291. MR 31 \#3475.

3. —_, On semi-hereditary rings, J. Math. Soc. Japan 13 (1961), 109-119. MR 25 \#2107.

4. R. W. Gilmer, Multiplicative ideal theory, Queen's Papers in Pure and Appl. Math., no. 12, Queen's University, Kingston, Ont., 1968. MR 37 \#5198.

5. W. W. Smith, Projective ideals of finite type, Canad. J. Math. 21 (1969), 1057-1061. MR 40, 133.

6. W. V. Vasconcelos, On finitely generated flat modules, Trans. Amer. Math. Soc. 138 (1969), 505-512. MR 39 \#199.

Department of Mathematics, University of Kansas, Lawrence, Kansas 66044 